\title{
Experimental Studies on the Reactive Thrust of the Mobile Robot of Arbitrary Orientation
}

\author{
Mikhail Polishchuk ${ }^{1}$, Mikhail Tkach², Igor Parkhomey ${ }^{3}$, Juliy Boiko ${ }^{4}$, Oleksander Eromenko \\ ${ }^{1,2,3}$ Department of Technical Cybernetics, National Technical University of Ukraine "Igor Sikorsky Kyiv Polytechnic \\ Institute", Ukraine \\ ${ }^{4}$ Department of Telecommunications and Radio Engineering, Khmelnytsky National University, Ukraine \\ ${ }^{5}$ Department of Physics and Electrical Engineering, Khmelnytsky National University, Ukraine
}

\begin{tabular}{l} 
Article Info \\
\hline Article history: \\
Received Oct 4, 2019 \\
Revised Mar 29, 2020 \\
Accepted May 5, 2020 \\
\hline
\end{tabular}

Keywords:

Mobile robot

Walking mechanisms

Climber robot

Jet thrust generator

\begin{abstract}
The problem of creating mobile robots of arbitrary orientation in the technological space is to ensure reliable retention of robots on the surface of any orientation. Therefore, well-known experimental studies are mainly devoted to the creation of systems for coupling the robot to the surface along which it moves. The purpose of this study is to create a device for compensating the gravitational load of a mobile robot. The article contains the results of experimental testing of a fundamentally new approach to counteract the gravitational load of a mobile robot, namely, the expediency of equipping the robot with a source of reactive thrust of a non-chemical origin. A pneumatic generator of aerodynamic lift is proposed as such a source. Such a force partially compensates or completely overcomes the gravitational load, while not allowing the transformation of a mobile robot into an aircraft. The specified condition is necessary to perform contact power technological operations in the maintenance of various industrial facilities. In other words, the thrust force should not exceed the adhesion forces of the mobile robot to the displacement surface. As a research method, a full factorial experiment of the operation of a jet thrust generator was used, which is a new way to increase the reliability of holding the robot on an arbitrary surface. The article describes the methodology and description of the full factorial experiment with varying independent factors at two extreme levels. As a result, an experimental solution to the problem of finding the quasi-optimal values of the aerodynamic lifting force depending on the parameters of the jet thrust generator is obtained. As a result, the combination of a new robot design with the results of experimental studies confirms the feasibility of using a pneumatic jet thrust generator as a means of increasing the reliability of holding mobile robots on an arbitrary orientation surface in the technological space.
\end{abstract}

Copyright $@ 2020$ Institute of Advanced Engineering and Science. All rights reserved.

\section{Corresponding Author:}

Juliy Boiko,

Department of Telecommunications and Radio Engineering,

Khmelnytsky National University,

11, Instytuts'ka str., Khmelnitsky, 29016, Ukraine.

Email: boiko_julius@ukr.net

\section{INTRODUCTION}

Mobile robot of vertical movement, also known as Climber Robot, are a new tool for mobile robotics. Robots of this type are able to perform production operations on surfaces of arbitrary orientation in the technological space while overcoming the gravitational load. The most effective application of these robots is the implementation of technological operations in extreme conditions of technological disasters, as well as the maintenance of high-rise buildings, industrial facilities, lines of high-voltage electrical systems, and even trees of forest and urban parklands.

In contrast to the well-known technical solutions of mobile robots of arbitrary orientation, this work proposes the use of aerodynamic lifting force as a means of counteracting the gravitational load of the robot. 
First, the task of setting up a complete factorial experiment was formulated in the article, the experimental procedure is described below, and analysis of variance and regression analysis of the experimental data is performed, including checking the obtained regression model for adequacy to the real process. At the end of the article, a study was made of the regression and analytical models of a pneumatic traction generator for mobile robots of arbitrary orientation in space. The practical application of the results of the above studies helps to increase the reliability of the retention of mobile robots on the displacement surface of arbitrary orientation in the technological space.

Most of the well-known studies in the field of creating mobile robots of arbitrary orientation are devoted to the improvement of the robot's adhesion systems to the surface on which it moves. This orientation is justified by the fact that the clutch system is the main factor in holding the mobile robot on a surface of arbitrary orientation in the technological space. So, for example, in [1-4], various means of coupling mobile robots to the surface along which it moves are proposed. These systems overcome the gravitational load for guaranteed retention of the robot on an arbitrarily oriented surface. The above technical solutions provide for the use of vacuum, electromagnetic, mechanical grips, as well as adhesion-based products. However, these technical solutions do not contain examples of the application of aerodynamic lifting force.

The system of holding the robot on the displacement surface as a vacuum generator was proposed in the design of the CCNY Robotics Lab City University New York robot [5]. An autonomous vacuum generator creates a zone of reduced pressure between the robot body and the surface along which the robot moves. This effect allows the mobile robot to compensate for the gravitational load, but only on flat surfaces of movement. The difference between the Boston Dynamics mobile robot is the presence of parallel pedipulators, which are equipped with needle mechanical hooks [6-8]. These hooks allow the robot to counteract the gravitational load when moving on the surface of the walls of tall buildings, and even on the surface of tree trunks. Mobile robot [9-12] also contains mechanical hooks for movement along tree trunks. Noting the originality of the designs of these models, it should be noted that the mechanical form of adhesion, as a means of counteracting the gravitational load, is not invariant to different surfaces of displacement of arbitrary orientation.

The electromagnetic system $[13,14]$ for holding a mobile robot on the surface on which it moves is characterized by high speed, simplicity of design, and relatively low power consumption [9, 10]. This robot clutch system can be effectively used for servicing large diameter metal pipelines, such as gas and oil pipelines. But electromagnetic grippers allow the robot to move only on ferromagnetic surfaces. Other methods of counteracting the gravitational load, for example, such systems of adhesion of mobile robots to the displacement surface, such as dry adhesion or electrical adhesion, are most effective from the point of view of energy conservation $[11,12]$. However, modern implementations of this type of adhesion to the displacement surface are characterized by an extremely low speed of movement of the robot due to the slowness of the adhesion process, which so far prevents their industrial use.

Studies [15-17] are devoted to modeling the dynamics of the movement of mobile robots and are aimed at reducing dynamic loads, which helps to increase the reliability of holding the mobile robot on the surface of motion. From this point of view, of interest the methodological approach to modeling the adhesion conditions of a mobile robot with a surface of arbitrary orientation, proposed in [18-22]. The method is based on the theory of resistance of materials and involves the calculation of normal stress values in the zone of contact of the robot with the surface on which the robot moves. This model allows you to control the grip strength of the legs of the robot with the surface on which it moves. However, these models do not contain solutions aimed at countering the gravitational load of a mobile robot, regardless of the physical and mechanical properties of the moving surface of mobile robots of arbitrary orientation in the technological space.

The above analysis of well-known publications shows that none of the studies discussed above has conducted experimental testing of the use of reactive thrust as a means of counteracting the gravitational load of a mobile robot. Thus, the task of experimental testing the application of the aerodynamic lift generator for mobile robots of arbitrary orientation is relevant.

The aim of the study below is to experimentally test the rationality of using a pneumatic jet thrust generator in the form of a means of compensating the gravitational load of a mobile robot. The main contribution of these studies are analytical expressions for calculating the quasi-optimal values urface of arbitrary orientation in the technological space. The results of the study can improve the efficiency of the operation of mobile robots of this type in various fields of industry.

\section{RESEARCH METHOD}

As a research method, the method of complete factorial extreme experiment was chosen. The difference between this method and other experimental research methods consists in the fact that the selected 
factors (variables) change at two extreme levels, namely: max and min. Therefore, this experiment is called extreme. The function of the studied variables is an incomplete quadratic polynomial, and the number of experiments is equal to $N=n^{k} \cdot m$, where: $n$ - is the number of levels of variation of factors; $k$ - is the number of factors; $m$ - is the number of repeated implementations of each experiment for good repeatability of the results of experiments and to reduce the variance of measurement errors.

Due to the lack of research on a pneumatic jet thrust generator as a means of counteracting the gravitational load of a mobile robot, a complete factorial experiment is necessary. In our case, the following number of experiments was performed: $N=2^{3} \cdot 8=64$ in accordance with the above formula. This experiment will allow not only to verify the possibility of using pneumatic jet thrust for mobile robots, but also to find the optimal or quasi-optimal values of the factors determining the efficiency of the pneumatic thrust generator.

\subsection{Solution of the problem under consideration}

For a better understanding of the proposed study, we will initially consider the technical solution of a mobile robot with a pneumatic jet thrust generator (Figure 1). The robot moves using walking mechanisms in the form of flexible pedipulators, at the ends of which vacuum gripping devices are installed to hold the robot on a surface of arbitrary orientation in space. The execution of the grips can be different, for example mechanical or electromagnetic, which in this case is not of fundamental importance. The main difference of the robot is the installation of a jet thrust generator with a pneumatic compressor on the Cardan suspension, which creates an aerodynamic force R. It is this force that counteracts the gravitational load $G=M g$ (where $M$ is the mass of the robot; $g$ is the gravitational acceleration).

The thrust generator $R$ is mounted on a gimbals in the XYZ coordinate system with three degrees of freedom and rotation angles $\alpha_{x}, \alpha_{y}, \alpha_{z}$. This technical solution allows, for any orientation of the robot relative to the horizon, to ensure that the line of action of the reactive thrust coincides with the line of action of the gravitational load $\mathrm{G}$, but these forces are always directed opposite to each other. Jet thrust should not exceed the force of adhesion of the robot to the moving surface so that the robot does not turn into an aircraft. Compensation of the gravitational load using a jet thrust generator provides increased reliability of the retention of a mobile robot on a surface of arbitrary orientation during technological operations. Flexible robot pedipulators are driven by mechanical reduction servo drives controlled by a controller. In addition, the robot is equipped with a power supply and a pneumatic unit to control the compressor of the traction generator.

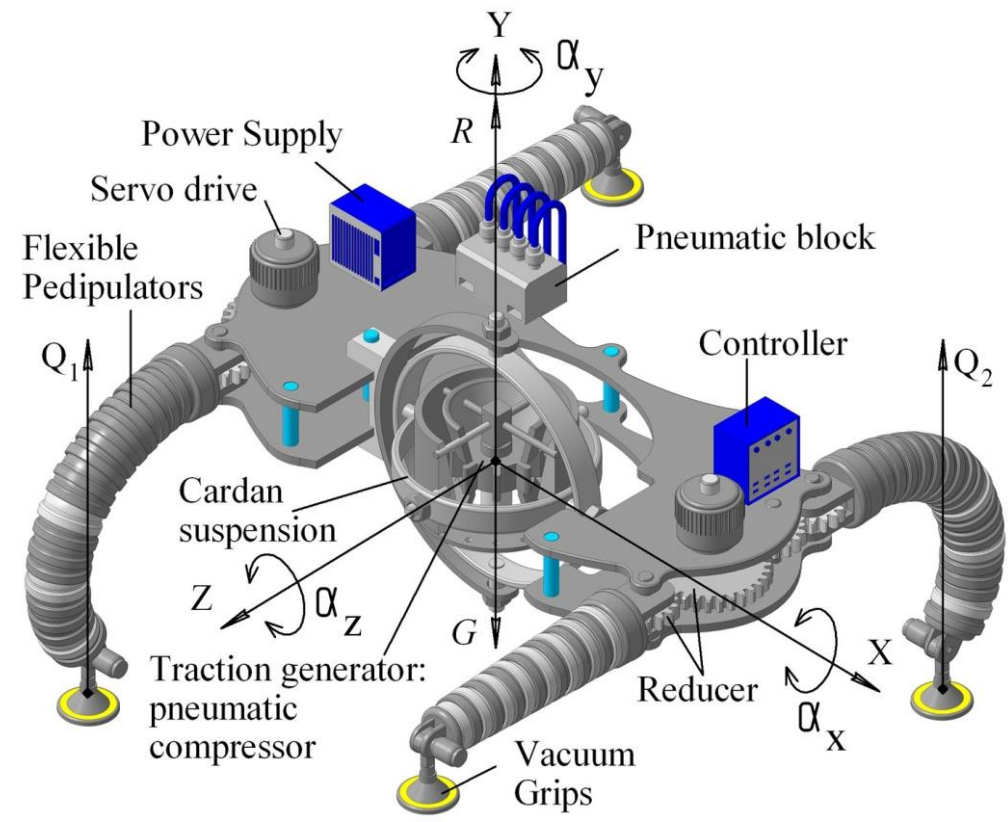

Figure 1. Mobile robot with pneumatic generator of aerodynamic lift

At the contact points of the robot's pedipulators with the displacement surface, forces of normal reactions $Q_{1}$ and $Q_{2}$, which are the result of the influence of the weight of the robot on the surface on which the robot moves (Figure 1) when the robot stands on only two legs. From the equilibrium equations of all the forces that act on the robot, graphical dependences $Q_{1}=f(\alpha)$ and $Q_{2}=f(\alpha)$ of the reaction forces and on the angle $\alpha$ of the inclination of the robot to the horizon are obtained Figure 2. 


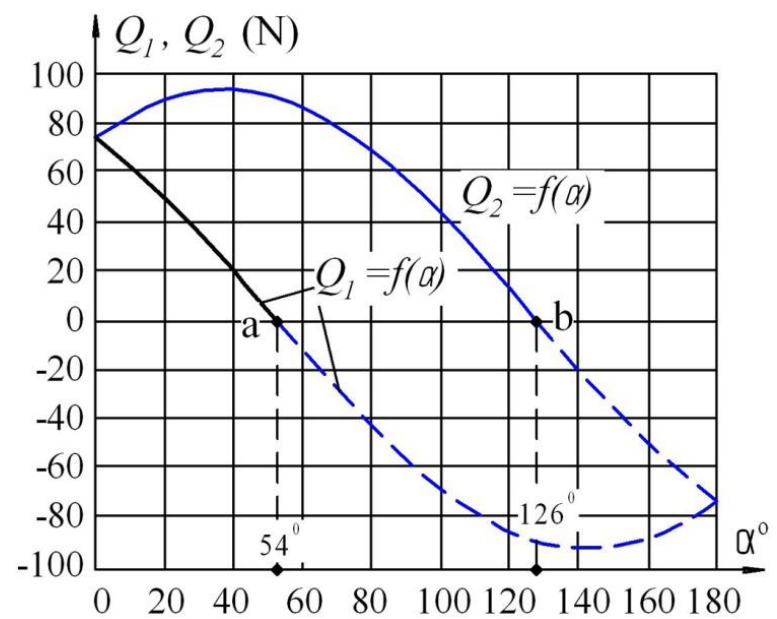

Figure 2. Change in reaction forces $Q_{i}=f(\alpha)$ from the angle $\alpha$ of the tilt of the robot to the horizon

The indicated reaction forces characterize the adhesion forces of the robot with the displacement surface. The graphs show that at different angles of inclination of the robot to the horizon, these forces have different values. When the values of forces $Q_{1}$ and $Q_{2}$ reach zero, then the legs of the robot begin to slip. This condition is dangerous because the mobile robot can separate from the moving surface. Therefore, when the first leg begins to slide, for example, at point "a" in Figure 2, that is $Q_{1}=0, \alpha \approx 54^{\circ}$ when, it is necessary to turn on the reactive traction generator to compensate for the gravitational load. The second leg will begin to slip only at point " $b$ " $\alpha \approx 126^{\circ}$ when, because it experiences less load due to the tilt of the robot to the horizon. That is why the controller (Figure 1) regulates the performance of the traction generator at various angles of inclination of the mobile robot relative to the horizon. The operation of the robot is described in more detail in [23-27]. Here we turn to experimental studies of the jet thrust generator.

The results of kinematic and dynamic analysis of a mobile robot with a pneumatic jet thrust generator, described in [5,16], allowed us to carry out an a priori analysis of the influence of the parameters of a pneumatic jet thrust generator on aerodynamic lift. However, these dependencies do not take into account the stochastic effect of the thrust generator parameters on the value of jet thrust, and most importantly, like any analytical model, they cannot serve as experimental testing of the use of jet thrust generator for mobile robots.

As is known in the formulation of factorial experiments, the function of the studied variables is an incomplete quadratic polynomial [20, 28-32], and the number of experiments is equal to, $N=n^{k} \cdot m$ where: $n$ is the number of levels of variation of factors; $k$ is the number of factors; $m$ is the number of repeated implementations of each experiment for good repeatability of the results of the experiments and reduce the variance of the measurement errors. In our case, it is necessary to conduct an $N=2^{3} \cdot 8=64$ experiment. To conduct research, an experimental stand was created, the circuit diagram of which is shown in Figure 3, and the general view in Figure 4. The stand includes a source of increased pressure (up to 8 bar) of compressed air in the form of compressor 1. This compressor through the air preparation unit 2, pressure gauge 3 and pneumatic distributor 4 creates increased pressure in chamber " $\mathrm{A}$ " of the draft generator 5 , which is measured by pressure gauge 6 . Compressed air flowing out through many nozzles 9 creates jet thrust, as a result of which thrust generator 5 moves in the guides of the translational kinematic pair 7 mounted on a fixed base 8 . The force of the generated thrust is measured digitally dynamometer 10 .

An external power load on the thrust generator 5 was created by a pneumatic cylinder 11 mounted on an arm 12. Compressed air was supplied to the power cylinder by means of an electromagnetic pneumatic distributor 13 controlled by a controller 14. In Figure 4 shows a general view of an experimental stand with a pneumatic traction generator for a mobile robot. As can be seen in the photo, the piston compressor 2 was used as a source of overpressure in the chamber of the thrust generator 1, which developed a pressure of up to 8 bar with a compressed air supply of $150 \mathrm{l} / \mathrm{min}$. Pressure Control was carried out by pressure gauge 3, and the pressure value was set by pressure regulator 4 in the range $(2 \ldots 8)$ bar.

Creating an external load, which includes the weight of the robot, on the traction generator was created by a pneumatic cylinder 5 connected to an analog displacement sensor 6 and controlled by a positioning controller 7 using the control panel 8. The pneumatic traction force was measured with a digital dynamometer 9 with a force of up to $50 \mathrm{~kg}(490 \mathrm{~N})$ with a measurement error of $\pm 5 \mathrm{~g}$ with a force of $0 \ldots 10$ $\mathrm{kg}$ and $\pm 10 \mathrm{~g}$ in the range of forces of $10 \ldots 45 \mathrm{~kg}$. 


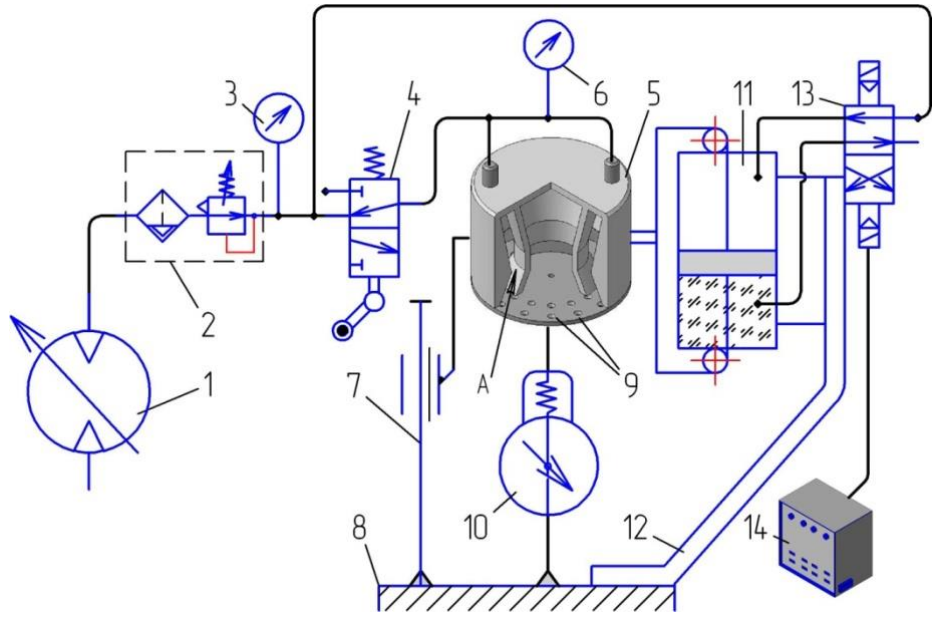

Figure 3. Schematic diagram of the experimental stand

During the experiment, compressed air was supplied from the compressor to the thrust generator 1 (Figure 5), mounted on the rings 2 of the Cardan suspension, through fittings 3. As a result, a zone of increased pressure was created in chamber " $A$ " in the range $(2 \ldots 8)$ bar. Compressed air, leaving through the nozzle 4 of the membrane 5, created a jet thrust. Since the conditions for conducting a full factorial experiment provide for the variation of factors at extreme levels of min and max, these membranes changed according to the values of nozzle diameters $d=\min \ldots \max$ and their number $n=\min \ldots \max$, including the average level $d_{s}, n_{s}$ of values experimental matrix.

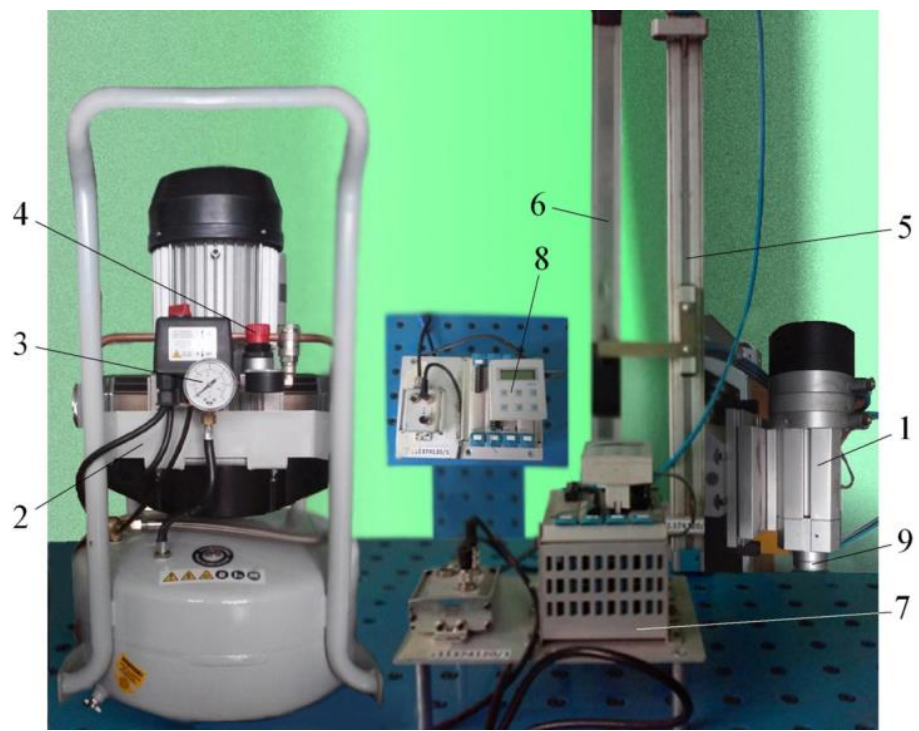

Figure 4. The experimental stand of the pneumatic traction generator

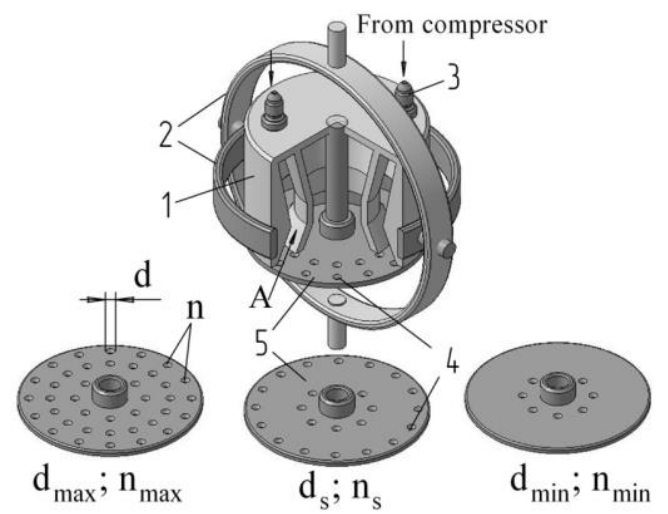

Figure 5. Pneumatic traction generator with replaceable membranes 
As a hypothesis, which requires further confirmation, at the stage of problem statement, we present the objective function in the form of a formula

$$
R=C_{0} f_{1}(n) f_{2}\left(p_{j}\right) f_{3}\left(d_{k}\right) \rightarrow \max
$$

With restrictions:

$$
\begin{aligned}
& \frac{\pi d^{2}}{4} n_{\min } \leq F_{i}(d, n) \leq\left(\frac{\pi d^{2}}{4} n_{\max }\right) \\
& p_{\min }<p_{j} \leq p_{\max } \\
& d_{\min } \leq d_{k} \leq d_{\max }
\end{aligned}
$$

Where $R$ - aerodynamic jet thrust, $N ; C_{0}$ a constant coefficient taking into account the influence of external influences that are not displayed in the objective function; $f_{1}\left(n_{i}\right), f_{2}\left(p_{j}\right), f_{3}\left(d_{k}\right)$ - factor dependencies (variable functions) to be determined; $F$ is the nozzle cross-sectional area, determined by the diameter $d(\mathrm{~m})$ of the gas outlets and their number $n ; p_{j}$ - pressure in the nozzles of the traction generator, (bar).

The first of these restrictions (2) for the factors of function (1) indicates that the number of nozzles for gas outflow should be sufficient to create reactive lifting force. The second limitation is dictated by the international standard for modern pneumatic systems in the pressure range $p_{i}=(1 \ldots 10) \cdot 10^{5} \mathrm{~Pa}$. The third restriction (2) determines a sufficient range of the diameter $\mathrm{d}$ of the holes for the gas to flow in the jet thrust generator. In accordance with the accepted restrictions (2), when setting up a full-factor experiment, the variation of the objective function factors was carried out in the following limits:

parameters in the first use, especially under the equation. The sample of equation is as follow:

$10 \leq n_{i} \leq 80$

$\left(1 \leq p_{j} \leq 8\right) 10^{5} \mathrm{~Pa}$

$\left(0,001 \leq d_{k} \leq 0,0045\right) \mathrm{m}$

Where $n_{\mathrm{i}}$ is the number of nozzles; $p_{j}$ - pressure in nozzles with a diameter $d_{k}$.

A total of $N=n^{k} \cdot m=2^{3} \cdot 8=64$ experiments was conducted, where: $n$ - is the number of levels of variation of factors (max, $\min ) ; k$ - is the number of factors; $\mathrm{m}$ is the number of repeated implementations of each experiment for good repeatability of the results of the experiments and reduce the variance of the measurement errors.

When setting up factor experiments, coded values of variables are used. This greatly facilitates not only the calculation of constant coefficients, but also the study of the subsequently obtained regression model of the response function. Therefore, we pass from the coordinate system of the variables $n, p, d$ in the natural dimension (3) to a dimensionless coordinate system $x_{1}, x_{2}, x_{3}$. Using the formula [23], we transform the independent variables into dimensionless variables:

$$
x_{i}=\frac{2\left(\ln x_{i}-\ln x_{i \max }\right)}{\ln x_{i \max }-\ln x_{i \min }}+1
$$

Where $x_{i}$ is the natural dimension of factors. In the dimensionless coordinate system for the experiment model $n^{k}=2^{3}=8$, the upper level corresponds to the value of the factor " +1 " ( $\max$ ), the lower level " -1 " ( $\mathrm{min})$, and the coordinates of the center of the experimental design coincide with the origin of the coordinate system.

The coding results of the upper and lower levels of variation of factors are summarized in Table 1.

Table 1. Factor Change Levels

\begin{tabular}{ccccccc}
\hline Levels of & $n$ & $n$ & $p$, bar & $p$, bar & $d, m$ & $x_{3}$ \\
factors & $x_{1}$ & $\ln x_{1}$ & $x_{2}$ & $\ln x_{2}$ & $-m$ \\
\hline Upper +1 & 80 & 4.38 & 8 & 2.08 & 0.0045 & -5.40 \\
Main 0 & 45 & - & 4.5 & - & 0.00275 & - \\
Lower -1 & 10 & 2.3 & 2 & 0.69 & 0.001 & -6.91 \\
\hline
\end{tabular}

From Table 1 we find dimensionless variables:

$$
\begin{aligned}
& x_{1}=\frac{2\left(\ln x_{1}-4,38\right)}{4,38-2,3}+1=0,95 \ln x_{1}-3,19 ; \\
& x_{2}=\frac{2\left(\ln x_{2}-2,08\right)}{2,08-0,69}+1=1,44 \ln x_{2}-1,99 ; \\
& x_{3}=\frac{2\left(\ln x_{2}+5,40\right)}{-5,40+6,91}+1=1,32 \ln x_{3}+8,15 .
\end{aligned}
$$


The model $n^{k}=2^{3}=8$ of a complete factorial experiment is presented in the form of an incomplete quadratic polynomial, which can be written taking into account the interaction of factors and in coded variables

$$
y=b_{0}+b_{1} x_{1}+b_{2} x_{2}+b_{3} x_{3}+b_{12} x_{1} x_{2}+b_{13} x_{1} x_{3}+b_{23} x_{2} x_{3}+b_{123} x_{1} x_{2} x_{3}
$$

Where $b_{0}, b_{i, j, k}$ constant factors for factors: $x_{i, j, k} ; i, j, k=1,2,3$

In the Table 2 show the levels of variation of independent factors and their interaction. This table displays the planning matrix of the full factorial experiment. According to the given matrix, each of the factors was varied: $n$-number of nozzle openings, $p$ - pressure in nozzles of diameter $d$ at two extreme levels of "+1" (max) and " -1 " (min).

Table 2. Extreme Experiment Planning Matrix

\begin{tabular}{|c|c|c|c|c|}
\hline \multicolumn{5}{|c|}{ Factors and levels of their variation } \\
\hline \multirow{2}{*}{$\begin{array}{c}\text { № } \\
\text { experience }\end{array}$} & \multirow{2}{*}{$\begin{array}{c}x_{0} \\
\text { The code }\end{array}$} & $x_{1}$ & $x_{2}$ & \multirow{2}{*}{$\begin{array}{c}x_{3} \\
\text { The code } \quad d_{i}, \mathrm{~m}\end{array}$} \\
\hline & & The code $n_{i}$ & The code $p_{i}$, bar & \\
\hline 1 & + & +80 & +8 & $+0,0045$ \\
\hline 2 & + & 10 & + & 0,0045 \\
\hline 3 & + & +80 & - & 0,0045 \\
\hline 4 & + & 10 & - & 0,0045 \\
\hline 5 & + & 80 & + & 0,001 \\
\hline 6 & + & 10 & + & 0,001 \\
\hline 7 & + & 80 & - & 0,001 \\
\hline 8 & + & $-\quad 10$ & $-\quad 2$ & 0,001 \\
\hline \multicolumn{5}{|c|}{ Variation in the interaction of factors } \\
\hline $\begin{array}{c}\text { № } \\
\text { experience }\end{array}$ & $\begin{array}{c}x_{1} x_{2} \\
\text { The code }\end{array}$ & $\begin{array}{c}x_{1} x_{3} \\
\text { The code }\end{array}$ & $\begin{array}{c}x_{2} x_{3} \\
\text { The code }\end{array}$ & $\begin{array}{l}x_{1} x_{2} x_{3} \\
\text { The code }\end{array}$ \\
\hline 1 & + & + & + & + \\
\hline 2 & - & - & + & - \\
\hline 3 & - & + & - & - \\
\hline 4 & + & - & - & + \\
\hline 5 & + & - & - & - \\
\hline 6 & - & + & - & + \\
\hline 7 & - & - & + & + \\
\hline 8 & + & + & + & - \\
\hline
\end{tabular}

In the Table 3 shows the results of experimental studies of the influence of the parameters of a pneumatic generator on the value of jet thrust $R$. Vertical columns show the obtained value of jet thrust, and horizontal rows contain the results of the repetition of each experiment. The column $\overline{R_{i}}$ gives the average values of jet thrust. In the Table 4 shows the encoded values (logarithms) of jet thrust and dispersion of experimental results.

Table 3. Experiment Results

\begin{tabular}{ccccccccccc}
\hline № & \multicolumn{1}{c}{ Jet thrust $R$ (with the number of experiments $\left.m \rightarrow R_{1} \ldots R_{8}\right)$} & \multicolumn{4}{c}{ Averages } \\
experience & $R_{1}$ & $R_{2}$ & $R_{3}$ & $R_{4}$ & $R_{5}$ & $R_{6}$ & $R_{7}$ & $R_{8}$ & $\bar{R}_{i}$ \\
\hline 1 & 828 & 745 & 806 & 780 & 760 & 802 & 805 & 820 & 793 \\
2 & 104 & 93 & 98 & 102 & 96 & 103 & 98 & 97 & 99 \\
3 & 216 & 210 & 209 & 212 & 196 & 215 & 212 & 198 & 209 \\
4 & 27 & 25 & 26 & 24 & 28 & 24 & 26 & 25 & 26 \\
5 & 184 & 178 & 172 & 176 & 180 & 182 & 180 & 178 & 179 \\
6 & 23 & 21 & 23 & 24 & 22 & 23 & 20 & 23 & 22 \\
7 & 48 & 44 & 45 & 47 & 46 & 47 & 45 & 46 & 46 \\
8 & 6 & 5,8 & 5,5 & 6 & 5,4 & 6 & 6 & 5,6 & 5,8 \\
\hline
\end{tabular}

The data obtained make it possible to determine the numerical values of the constant coefficients in equation (6). Due to the fact that the full factorial experiment relates to orthogonal plans, the calculation of regression coefficients is greatly simplified. According to the formula $[18,19]$ they can be defined as:

$$
b_{i}=\frac{\sum_{i=1}^{n} x_{i} \bar{y}_{i}}{m}
$$

Where $i-$ is the experience number; $\mathrm{m}$ is the number of test points in the plan; $\bar{y}_{i}-$ the average response of function (6) by the number of repeated experiments at the corresponding point in the matrix of the experimental design.

According to the theory of a full factorial experiment, at $i=0$, the coefficient $b_{0}$ is calculated. When each factor varies at two levels $(+1)$ and $(-1)$, the calculations are reduced to assigning to the column of signs $(+,-)$ the column of the corresponding factor and algebraic addition of the obtained values. Subsequent 
dividing the result by the number of experimental points $(n=8)$ in terms of the experiment gives the desired coefficient: $b_{0}=4,22 ; b_{1}=1,04 ; b_{2}=0,67 ; b_{3}=0,75 ; b_{12}=0,005 ; b_{13}=0 ; b_{23}=-0,0025 ; b_{123}=-0,0025$.

Table 4. Coded Values and Variance of Test Results

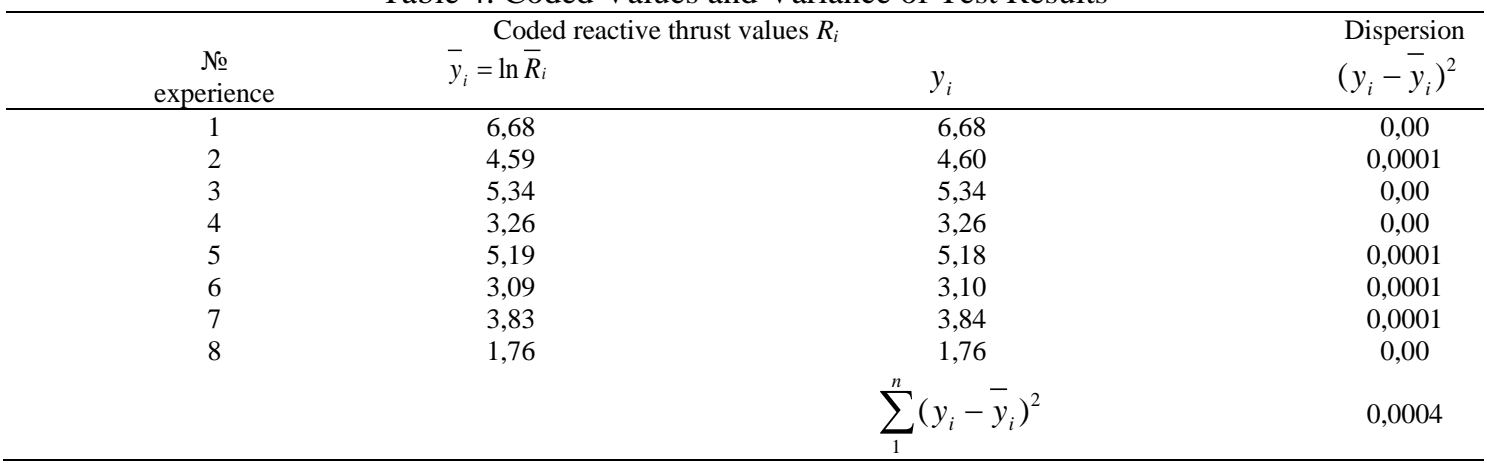
equation:

The values of these coefficients are substituted into equation (6) and we obtain the regression

$$
y=4,22+1,04 x_{1}+0,67 x_{2}+0,75 x_{3}+0,005 x_{1} x_{2}-0,0025 x_{2} x_{3}-0,0025 x_{1} x_{2} x_{3}
$$

Before using this equation as a regression model of the response function, it must be subjected to statistical verification, the contents of which are described below.

\subsection{Regression analysis}

According to the requirements of the regression analysis, it is necessary to check the homogeneity of the variances of the experimental data of the Table 3. Since the theoretical values of the variances are unknown, we verify their homogeneity on the basis of statistical estimates of the obtained empirical material, for which we determine the variance of the it, experiment using the formula [23-26]:

$$
S_{i}^{2}=\frac{1}{m-1} \sum_{k=1}^{m}\left(y_{i k}-\bar{y}_{i}\right)^{2}, \quad \mathrm{i}=\overline{1, n} ; \quad \mathrm{k}=\overline{1, m},
$$

According to the results of calculations by formula (9), it was determined: the maximum value of the variances $S_{i}^{2}=0,0039$; sum of all variances $\sum_{i=1}^{n} S_{i}^{2}=0,0147$ and dispersion of repetition of experiments $S_{m, n}^{2}=\frac{1}{n} \sum_{i=1}^{n} S_{i}^{2}=0,0018$.

The homogeneity of the variances is checked by the Kochren criterion, as the ratio of the maximum line-by-line variance to the sum of all variances:

$$
G=\frac{S_{\max }^{2}}{\sum_{i=1}^{n} S_{i}^{2}}=\frac{0,0039}{0,0147}=0,2653
$$

To test the hypothesis of homogeneity of dispersions, we use the data from the table [23], which shows the numerical values of the Kochren criterion. This table allows you to compare the ratio of the maximum row dispersion to the sum of all variances. In this case, we compare the calculated value (10) obtained with its allowable critical ratio.

Having taken the significance level (usually $q=0.05$ ) and determined the number of degrees of freedom: $f_{1}=m-1=7 ; f_{1}=n-1=8$, according to the Table [23] we find the critical ratio $G_{\kappa p}=0,4137$ and compare it with the calculated value $G=0,2653<G_{\kappa p}=0,4137$. The result of this comparison allows us to accept the hypothesis of homogeneity of variances. This, in turn, makes it possible to use the empirical material of the Table 3 to obtain an approximating model of the response function.

The significance of the coefficients of the regression equation (8) is checked using the t-student criterion. To do this, we find the variance of the error in determining the coefficients [15]:

$$
S_{b_{1}}^{2}=\frac{1}{m n} S_{m n}^{2}=\frac{0,0018}{64}=0,000028125, \quad S_{b}=0,0053
$$

Next, for each coefficient of equation (8), we determine the calculated value of the Student criterion as the ratio of the absolute value of the regression coefficient to the standard deviation of the error of its determination: $t_{\mathrm{i}}=\left|b_{\mathrm{i}}\right| / S_{b i}, t_{0}=796 ; t_{1}=196 ; t_{2}=126 ; t_{3}=143 ; t_{12}=0,94 ; t_{23}=0,47 ; t_{123}=0,047$, where the indices of the criteria correspond to the indices of the coefficients of the regression equation (8).

To find the critical value of tcr according to the table of the appendix $[13,14]$ with the number of degrees of freedom $f^{*}=n(m-1)=56$ and with a significance level of $q=0.05$, we find the critical value $t_{c r}=2$ of student criterion. The regression coefficients are significant provided that $\left.t_{i}\right\rangle t_{c r}$. Comparing the calculated 
values of the criterion $t_{i}$ with the tabular $t_{c r}=2$, we conclude that it is necessary to exclude from the equation (8) the coefficients of pair interaction of factors $x_{12}, x_{23}$ and triple interaction $x_{123}$, as statistically insignificant. There is no need to recalculate the remaining coefficients, since in the orthogonal design of the experiment all the coefficients are independent.

Taking into account the obtained calculations, we write equation (8) in the following form:

$$
y=4,22+1,04 x_{1}+0,67 x_{2}+0,75 x_{3}
$$

Before proceeding to the analysis of the obtained regression model of the objective function (1) in the form of equation (12), it is necessary to verify the adequacy of the model to the experimental data by assessing the deviations of the values (Table 4) from the experimentally established ones and averaged over the number of parallel experiments at points of the factor space. To verify the adequacy of model (12) to the real process of functioning of the traction generator, we use the well-known Fisher criterion:

$$
S_{a}^{2}=\frac{m}{n-k} \sum_{i=1}^{8}\left(\bar{y}_{i}-y_{i}\right)^{2}=0,0008,
$$

Where $\hat{y}_{i}$ is the value of the response function, determined by equation (12) for each experiment; $k=4$ is the number of members of the approximating polynomial in the form of the specified equation. Then the calculated value of the $F$-criterion or dispersion ratio will be [21]:

$$
F=S_{a}^{2} / S_{m, n}^{2}=0,0008 / 0,0018=0,444
$$

In our case, the number of degrees of freedom is: $f_{1}=n-k=4 ; f_{2}=n(m-1)=56$. According to the table [23] and at the significance level $q=0.05$, we determine the critical value of the parameter $F_{\kappa p}=2,56$. According to the methodology of the factor experiment, the ratio of the model is recognized as adequate to the real process. The comparison result $F=0,571<F_{\kappa p}=2,56$ allows us to establish the adequacy of the regression equation (12) to the real process of gas outflow in the thrust generator, depending on the number " $n$ " of nozzles, the diameters " $d$ " of the openings of their cross section and the pressure " $p$ " in the thrust generator.

To assess the contribution of the regression coefficients to the equation of model (12), we use the multiple correlation coefficient, the calculation result of which according to the Table 5 amounted to:

$$
K=\sqrt{1-\sum_{i=1}^{n}\left(\bar{y}_{i}-y_{i}\right)^{2} / \sum_{i=1}^{n}\left(\bar{y}_{i}-\bar{y}\right)^{2}}=0,99998 .
$$

The correlation coefficient can vary within the limits $0 \leq K \leq 1$ where equality to the lower limit indicates the absence of any contribution made by the regression coefficient, and, on the contrary, equality to the upper limit indicates that the regression equation fully describes the experimental results. In our case, taking into account the data in Table 5, the correlation coefficient was $K=0.9998$, therefore, the regression equation (12) almost completely describes the experimental results.

Table 5. Calculation of the correlation coefficient

\begin{tabular}{cccc}
\hline $\begin{array}{c}\text { № } \\
\text { experience }\end{array}$ & $\bar{y}_{i}=\ln t_{i}$ & $\left(\bar{y}_{i}-\bar{y}\right)$ & $\left(\bar{y}_{i}-\bar{y}\right)^{2}$ \\
\hline 1 & 6,68 & 2,46 & 6,05 \\
2 & 4,59 & 0,37 & 0,14 \\
3 & 5,34 & 1,12 & 1,25 \\
4 & 3,26 & $-0,96$ & 0,92 \\
5 & 5,19 & 0,97 & 0,94 \\
6 & 3,09 & $-1,13$ & 1,28 \\
7 & 3,83 & $-0,39$ & 0,15 \\
8 & 1,76 & $-2,46$ & 6,05 \\
\hline$n$ & $\bar{y}=4,218$ & & $\sum\left(\bar{y}_{i}-\bar{y}\right)^{2}=16,78$ \\
& & & $\sum\left(\bar{y}_{i}-y\right)^{2}=0,0004$ \\
\hline
\end{tabular}

\section{RESULTS AND DISCUSSION}

Known systems for adhesion mobile robots to a surface of arbitrary orientation in space are necessary to hold robots on the surface of their movement. Experimental studies of adhesion to the surface on which mobile robots move were carried out in the above works [10,13,14], but without the use of a jet thrust generator. In [32, 33], devices were proposed for compensating the gravitational load of mobile robots. But in these works, there are no experimental data or a numerical analysis of the quasi-optimal characteristics of a device for overcoming the gravitational load of a mobile robot. Thus, the proposed results of an experimental study are innovative. However, these systems are not invariant to surfaces with different physical and mechanical characteristics. 
In contrast to known systems, the use of aerodynamic force or pneumatic jet thrust does not depend on the properties of the surface of movement of a robot of arbitrary orientation. However, the aerodynamic force, as a means of compensating the gravitational load, should not exceed the force of adhesion of the robot to the displacement surface. This condition is necessary for the mobile robot to perform contact technological operations.

As a result of the experimental study, by setting up a complete factorial experiment, an empirical data array of the influence of the parameters of the pneumatic traction generator on the aerodynamic lift force was obtained as a means of counteracting the gravitational load of a mobile robot. The indicated empirical data array illustrates the stochastic effect of the thrust generator parameters on the aerodynamic force of maintaining a mobile robot on a surface of arbitrary orientation in the technological space.

Equation (12) in the encoded variables establishes a statistical dependence between the optimization parameter and factors, i.e. variable number of nozzles, the diameter of their cross section and pressure in them. By analyzing the magnitude and sign of each regression coefficient (12), one can obtain reliable information about the influence of factors on the optimization parameter and determine the search strategy for the extremum of the objective function. In equation (12), the coefficients for the variables are positive. This means that an increase in the number of nozzles in the traction generator and pressure in the nozzles leads to an increase in the aerodynamic lift of the traction generator [18-22]. The same effect also occurs with an increase in nozzle diameter. However, the number of nozzles in the pneumatic traction generator has a dominant effect.

To find the coordinates of the extremum, we verify the possibility of the existence of the latter in a given region of the factor space with restrictions (3). To this end, we substitute the coded values of variables (5) into equation (12) and, after potentiation, we obtain the desired expression of the objective function (1) in physical units of the factors:

$$
R=e^{5.68}(n)^{0.988}(p)^{0.965}(d)^{0.99} \rightarrow \max
$$

Where $e=2.72$ is a constant coefficient, the basis of natural logarithms.

The condition for the existence of an extremum of the objective function (15) at some point in the factor space with coordinates has the form:

$$
\frac{\partial R}{\partial n}\left(n_{0}\right)=\frac{\partial R}{\partial p}\left(p_{0}\right)=\frac{\partial R}{\partial d}\left(d_{0}\right)=0
$$

In a geometric interpretation, this condition should determine the equality of the angles of inclination of the tangents to the curve of the functional dependence at the same points $k_{n}\left(n_{0}, R\right)$ and $k_{d}\left(d_{0}, R\right)$; provided $p_{i}=p_{0}$. According to the experimental data, were obtained the dependences of the jet thrust $R$ on the diameter " $d$ " of the generator nozzles (Figure 6) and 3D graphs of the dependence of the jet thrust R on the simultaneous change the diameters " $d$ " of the nozzles and their number " $n$ " (Figure 7). However, as can be seen from the graphs of Figure 6 and Figure 7, the indicated angles of inclination of the tangents to the dependency paths are not equal. Therefore, condition (16) is not satisfied for the values of factors within the range of variation. In this case, the optimization problem can be solved analytically as a problem on the conditional extremum of the objective function by finding the extreme values of the optimization parameter at the boundaries of the factor space. In this case, we are interested in the maximum possible value of the thrust force $R$ within the given limits (3) of variation of three independent factors: the number of nozzles " $n$ ", the overpressure " $p$ " in them and the diameters " $d$ " of the nozzle cross section.

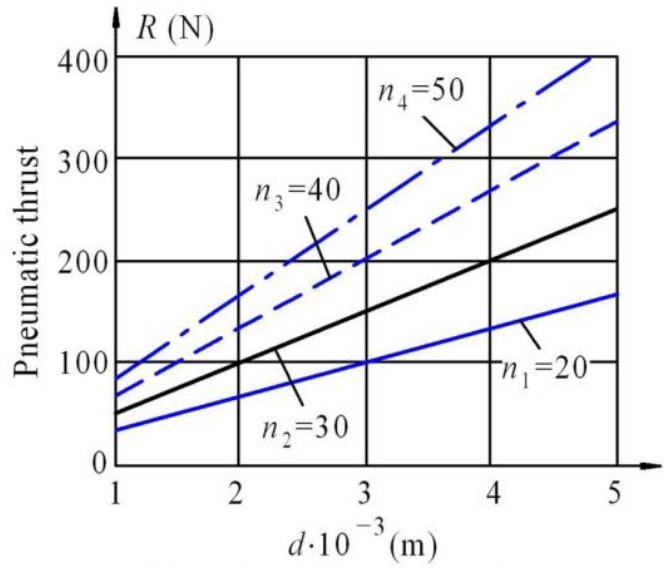

Diameters of nozzle openings

Figure 6. The dependence of the thrust force $R$ on the diameters $d$ of the holes and the number $n$ of nozzles at a pressure of $p=6$ bar 


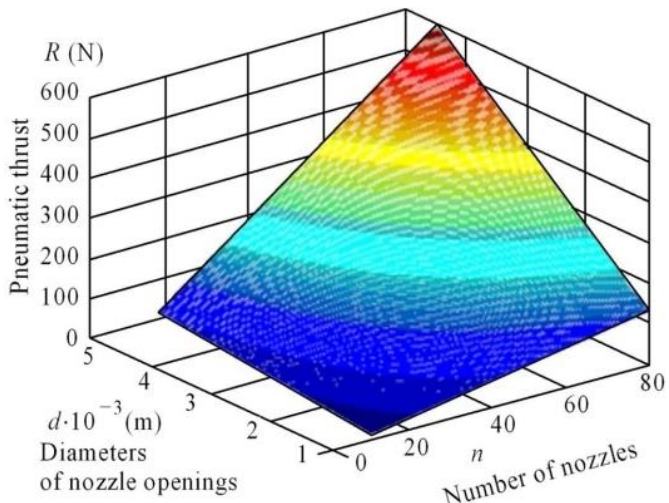

Figure 7. 3D graph of the thrust force $R$ as a function of the number of nozzles $\mathrm{n}$ and the diameters of their holes $\mathrm{d}$ at nozzle pressure $p=6 \mathrm{bar}$

To find the extreme values of the objective function (15), we find the partial derivatives with respect to each independent factor $(n, p, d)$ :

$$
\begin{aligned}
& \frac{\partial R}{\partial n}=0.988 e^{5.68}(n)^{-0.012}(p)^{0.965}(d)^{0.99} ; \\
& \frac{\partial R}{\partial p}=0.965 e^{5.68}(n)^{0.988}(p)^{-0.035}(d)^{0.99} ; \\
& \frac{\partial R}{\partial d}=0.99 e^{5.68}(n)^{0.988}(p)^{0.965}(d)^{-0.01} .
\end{aligned}
$$

It is seen from formulas (17) that the partial derivatives take positive values for all factors that are given by expressions (3). This means that the reactive thrust function $R$ increases with the growth of each factor and reaches its maximum value at the boundaries of the factor space, i.e., at the maximum values of the function factors (15):

$$
R_{\text {max }}=e^{5.68}\left(n_{\text {max }}\right)^{0.988}\left(p_{\text {max }}\right)^{0.965}\left(d_{\text {max }}\right)^{0.99}
$$

Thus, as a result of the experiment, it was found that the control of the thrust force $R$ can be carried out linearly depending on three independent factors: the number of nozzles " $n$ ", the overpressure " $p$ " in them and the diameters " $d$ " of the nozzle cross.

An analysis of the influence of the numerical characteristics of independent factors on the reactive thrust force is performed on the basis of the analytical expression (15) in physical measurement. In Figure 8 shows the graphs of the dependence of the thrust reactive force $R$ on the extreme values of independent factors: the number of nozzles $n_{\mathrm{min}}^{\max }$ and their diameters $d_{\mathrm{min}}^{\max }$ when the pressure in the nozzles changes in the experimental range $p=(2 \ldots 8)$ bar. According to the experimental planning matrix (Table 2), the extreme values of the factors were: number of nozzles $n_{\min }^{\max }=10 \ldots 80$, diameters $d_{\min }^{\max }=(1 \ldots 4,5) \cdot 10 \mathrm{E}-3 \mathrm{~m}$. In Figure 8 also shows the dependences of jet thrust $R$ on average values of factors, which, according to Table 3 represented: nozzles $n_{\mathrm{s}}=45$, and their diameters $d_{\mathrm{s}}=2,75 \cdot 10 \mathrm{E}-3 \mathrm{~m}$. These graphs confirm the dominant influence of the regression coefficient in equation (12) with the number of nozzles of the thrust generator and give a numerical characteristic of this dominant.

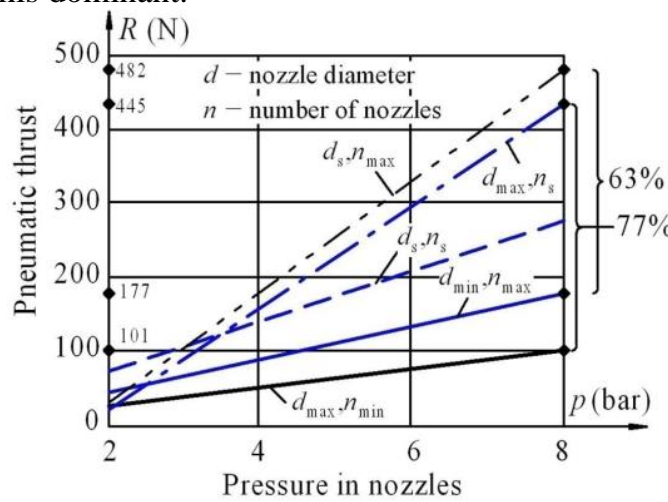

Figure 8. The dependence of the thrust force $R$ on the extreme values (min, max) of the number $n$ of nozzles and the diameters $d$ of the holes in the pressure range $p=(2 \ldots 8)$ bar 
Graphs Figure 8 show that at the maximum pressure $p_{\max }=8$ bar in the nozzles, when changing from the minimum value of their number $n_{\min }=10$ to average values $n_{s}=45$ and at maximum nozzle diameters $d_{\max }=45 \cdot 10 \mathrm{E}-3 \mathrm{~m}$, the increase in jet thrust is $77 \%$. While the transition from the minimum diameter of the nozzles $d_{\min }=1 \cdot 10 \mathrm{E}-3 \mathrm{~m}$ to the average values of the diameters $d_{s}=2,75 \cdot 10 \mathrm{E}-3 \mathrm{~m}$ at the maximum value of the number of nozzles $n_{\max }=80$, the indicated increase in jet thrust is less, namely $63 \%$. This means that the difference in the efficiency of controlling the number of nozzles and their diameters is $14 \%$. It should be noted that, taking into account the linear dependence of jet thrust on changeable factors, namely the number of nozzles, pressure in the nozzles and their diameters, this proportion will be observed for other pressure values in the nozzles.

The value of the absolute errors in the calculation of reactive thrust by the analytical dependence (15) relative to the experimental data (Table 3) was in the range from $0.7 \%$ to $5.1 \%$. Such deviations are acceptable in practice and provide an opportunity to assert the feasibility of using the aerodynamic force of the traction generator as a means of counteracting the gravitational load of a mobile robot of arbitrary orientation in the technological space when performing various production tasks.

\section{CONCLUSION}

Achieving the goal of the study, namely obtaining the regression function of the connection between the reactive thrust and such parameters as the number of nozzles, the diameters of the nozzles and the pressure in them proves the existence of conditional extreme at the boundaries of the factor space of changes in these parameters.

The obtained regression model of the dependence of aerodynamic lift on the parameters of the thrust generator in coded variables and its analytical model in natural units of measurement of independent factors can be used to design thrust generators as a means of counteracting the gravitational load. The adequacy of these models to the real process was verified by the Fisher criterion, and the significance of their influence coefficients was checked by the Student criterion.

In conclusion of these conclusions, it can be argued that an analysis of the numerical effect of the thrust generator parameters on the aerodynamic lift of a mobile robot confirms the dominant influence of the number of thrust generator nozzles.

The influence of the findings on the research area, namely, the study of the parameters of the pneumatic jet thrust generator, is that the experimental study provides sufficient grounds for asserting the feasibility of using aerodynamic force as a means of compensating and counteracting the gravitational load in order to increase the reliability of holding mobile robots in the technological space arbitrary orientation.

\section{REFERENCES}

[1] F. M. Silva and J. A. T. Machado. A Survey of Technologies and Applications for Climbing Robots Locomotion and Adhesion. London: Climbing and Walking Robots, 2010.

[2] M. Tanaka, M. Nakajima, Y. Suzuki and K. Tanaka, "Development and Control of Articulated Mobile Robot for Climbing Steep Stairs," IEEE/ASME Transactions on Mechatronics, vol. 23, iss. 2, pp. $531-$ $541,2018$.

[3] S. Sundarajoo, A. Shahrizan and A. Ghani, "Improvement of Auto-Tracking Mobile Robot based on HSI Color Model," Indonesian Journal of Electrical Engineering and Computer Science, vol.12, no. 3, pp. 1349-1357. 2018.

[4] K. Nagaoka, et al., "Passive Spine Gripper for Free-Climbing Robot in Extreme Terrain," IEEE Robotics and Automation Letters, vol. 3, iss.3, pp. 1765 - 1770, 2018.

[5] D.D. Raju and S.B. Jaju, "Developments in wall climbing robots: a review," International journal of engineering research and general science, №. 2, pp. 35, 2014.

[6] L. G. Lafaurie, F. E. Hoyos and J. E. Candelo-Becerra, "Mobile robot turned on by sound and directionally controlled by an external light source," International Journal of Electrical and Computer Engineering, vol.9, no 4, pp. 2840-2847, 2019.

[7] A. Azarfar, "Adaptive Fuzzy Control of Puma Robot Manipulator in Task Space with Unknown Dynamic and Uncertain Kinematic," Indonesian Journal of Electrical Engineering and Informatics, vol.6, no.4, pp. 458-470, 2018.

[8] F. G. Lopez, et al., "A predictive online path planning and optimization approach for cooperative mobile service robot navigation in industrial applications," in European Conference on Mobile Robots, 2017. ECMR 2017. pp. 1-6.

[9] A. Saundersa, D.I. Goldmanb, R.J. Fullb and M. Buehlera., "The RiSE Climbing Robot: Body and Leg Design," in Boston Dynamics, Unmanned Systems Technology, 2005. SPIE 2005, vol. 6230. pp. 13.

[10] T. L. Lam and Y. Xu. Tree Climbing Robot: Design, Kinematics and Motion Planning. New York, NY: Springer Heidelberg, 2012.

[11] M. Malley, M. Rubenstein and R. Nagpal, "Flippy: A soft, autonomous climber with simple sensing and control," in International Conference on Intelligent Robots and Systems, 2017. IROS 2017. IEEE/RSJ, 2017, pp. 6533 6540. 
[12] C. Menon, M. Murphy and M. Sitti, "Gecko Inspired Surface Climbing Robots," in International Conference on Robotics and Biomimetics, 2004, pp. 6.

[13] J.W. Beard, S.L. Canfield and D.A. Bryant. "Climbing vehicle with suspension mechanism." U.S. Patent 9428 231, Aug. 30, 2016.

[14] S.F. Yatsun, V. Ya. Mishchenko. and A.A. Cherepanov. "Transport device for movement on vertical metal surfaces." RF patent for utility model 101683, Jan. 27, 2011.

[15] M. Polishchuk and M. Tkach, "Mobile Robot with an Anthropomorphic Walking Device: Design and Simulation," FME Transactions, vol. 48, no 1, pp. 13-20, 2020.

[16] M. F. Silva, J. A. T. Machado, "New Technologies for Climbing Robots Adhesion to Surfaces", in International Workshop on New Trends on Science and Technology, 2008.

[17] M.N. Polishchuk and V.V. Oliinyk, "Dynamic Model of a Stepping Robot for Arbitrarily Oriented Surfaces," in International Conference on Computer Science, Engineering and Education Applications, 2019. ICCSEEA 2019. Advances in Computer Science for Engineering and Education II, 2019, pp. 32-42.

[18] Y. H. Chen, P. J. Lee and Y. C. Tsai, "Side robot - a wall climber implements in the autonomous monitor robot," in International Conference on Fuzzy Theory and Its Applications, 2017. iFUZZY 2017. pp. 1-4.

[19] H. Wang and A. Yamamoto, "Analyses and Solutions for the Buckling of Thin and Flexible Electrostatic Inchworm Climbing Robots," IEEE Transactions on Robotics, vol.33, iss. 4, pp. 889 - 900, 2017.

[20] I. Parkhomey, J. Boiko and O. Eromenko, "Identification information sensors of robot systems," Indonesian Journal of Electrical Engineering and Computer Science. vol.14, no. 3, pp. 1235-1243, 2019.

[21] S. Dong, "Gravity and inertial load adaptive control of wall-climbing robot," The Journal of Engineering, vol. 2019, iss. 13, pp. $442-446,2019$.

[22] Y. Guan, et al., "A Modular Biped Wall-Climbing Robot With High Mobility and Manipulating Function," IEEE/ASME Transactions on Mechatronics, vol. 18, iss. 6, pp. 1787 - 1798, 2012.

[23] I.I. Plyaskin, Optimization of technical solutions in mechanical engineering, Moskva M: Mechanical Engineering, 1982. 176p.

[24] P. A. Yvars, P. Lafon and L. Zimmer, "Optimization of mechanical system: Contribution of constraint satisfaction method," in IEEE International Conference on Computers \& Industrial Engineering, 2009. ICCIE 2009, 2009, pp. $1379-1384$

[25] A. N. Jati, et al., "A Multi-robot System Coordination Design and Analysis on Wall Follower Robot Group," International Journal of Electrical and Computer Engineering, vol.8, no. 6, pp. 5098-5106, 2018.

[26] N. Seegmiller and A. Kelly, "High-Fidelity Yet Fast Dynamic Models of Wheeled Mobile Robots," IEEE Transactions on Robotics, vol. 32, iss. 3, pp. 614-625, 2016.

[27] M. S. Noorani, "Hybrid Dynamical Model of a Gait Training Robot: Using Maggi's Method for Constrained Motions," in International Conference on Robotics and Mechatronics, 2018. IcRoM 2018. 2018 6th RSI, 2018, pp. $183-188$.

[28] P. M. Chu, et al., "Real-time 3D scene modeling using dynamic billboard for remote robot control systems," in 2017 IEEE International Conference on Multisensor Fusion and Integration for Intelligent Systems, MFI 2017. 2017 , pp. $354-358$.

[29] J. Boiko and O. Eromenko, "Signal Processing in Telecommunications with Forward Correction of Errors. Indonesian Journal of Electrical Engineering and Computer Science, vol.11 no 3, pp. 868-877, 2018.

[30] J. Itoh, et al., "Control Strategy for Starter Generator in UAV with Micro Jet Engine," in International Power Electronics Conference, IPEC-Niigata 2018. ECCE Asia, 2018, pp. 1567 - 1574.

[31] J. Boiko, V. Tolubko, O. Barabash, O. Eromenko and Y. Havrylko, "Signal processing with frequency and phase shift keying modulation in telecommunications," Telkomnika(Telecommunication Computing Electronics and Control), vol. 17, no 4, pp. 2025-2038, 2019.

[32] D. Ruffatto, et al., "Long-Duration Surface Anchoring With a Hybrid Electrostatic and Gecko-Inspired Adhesive," IEEE Robotics and Automation Letters, vol.3, iss. 4, pp. 4201 - 4208, 2018.

[33] X. Li. “Climbing robot Patent.” U.S. Patent 9738337, Aug. 22, 2017. 